\title{
Detection of bacterial contaminants and hybrid sequences in the genome of the kelp Saccharina japonica using Taxoblast
}

\author{
Simon M Dittami ${ }^{\text {Corresp., }} 1$, Erwan Corre ${ }^{2}$ \\ 1 UMR8227 - Sorbonne Universités CNRS UPMC, Station Biologique de Roscoff, Roscoff, Brittany, France \\ 2 FR2424 - Sorbonne Universités CNRS UPMC, Station Biologique de Roscoff, Roscoff, Brittany, France \\ Corresponding Author: Simon M Dittami \\ Email address: simon.dittami@gmail.com
}

Modern genome sequencing strategies are highly sensitive to contamination making the detection of foreign DNA sequences an important part of analysis pipelines. Here we use Taxoblast, a simple pipeline with a graphical user interface, for the post-assembly detection of contaminating sequences in the published genome of the kelp Saccharina japonica. Analyses were based on multiple blastn searches with short sequence fragments. They revealed a number of probable bacterial contaminations as well as hybrid scaffolds that contain both bacterial and algal sequences. This or similar types of analysis, in combination with manual curation, may thus constitute a useful complement to standard bioinformatics analyses prior to submission of genomic data to public repositories. Our analysis pipeline is open-source and freely available at http://sdittami.altervista.org/taxoblast and via SourceForge (https://sourceforge.net/projects/taxoblast). 


\section{DETECTION OF BACTERIAL CONTAMINANTS AND}

3 HYBRID SEQUENCES IN THE GENOME OF THE KELP

4 Saccharina Japonica USIng Taxoblast

5

6

7

8

Simon M. Dittami ${ }^{1 *}$ Erwan Corre $^{2}$

9

10

11

12

${ }^{1}$ Sorbonne Universités, UPMC Univ Paris 06, CNRS, UMR8227, Integrative Biology of Marine Models, 14 Station Biologique, CS 90074, 29688 Roscoff cedex, France. Email: simon.dittami@sb-roscoff.fr 15

${ }^{2}$ ABiMS platform, FR 2424 CNRS UPMC, Station Biologique, CS 90074, 29688 Roscoff cedex, France.

17

Email: erwan.corre@sb-roscoff.fr

18

19

*Corresponding author: phone +33 - 298292 362, fax +33 - 298292324

20 
21

22

23

24

25

26

27

28

29

30

31

32

33

34

35

36

37

38

39

40

41

42

43

44

45

46

47

48

49

50

51

52

53

54

55

56

57

58

\section{Abstract}

Modern genome sequencing strategies are highly sensitive to contamination making the detection of foreign DNA sequences an important part of analysis pipelines. Here we use Taxoblast, a simple pipeline with a graphical user interface, for the post-assembly detection of contaminating sequences in the published genome of the kelp Saccharina japonica. Analyses were based on multiple blastn searches with short sequence fragments. They revealed a number of probable bacterial contaminations as well as hybrid scaffolds that contain both bacterial and algal sequences. This or similar types of analysis, in combination with manual curation, may thus constitute a useful complement to standard bioinformatics analyses prior to submission of genomic data to public repositories. Our analysis pipeline is open-source and freely available at http://sdittami.altervista.org/taxoblast and via SourceForge (https://sourceforge.net/projects/taxoblast).

\section{Introduction}

Modern genome sequencing strategies rely strongly on the amplification of low quantities of Deoxyribonucleic acid (DNA), making them highly sensitive to even small contaminations in the samples. A study by Longo et al. (2011), for example, has shown almost $1 / 4^{\text {th }}$ of non-primate genomes available in the National Center for Biotechnology Information (NCBI) databases to be contaminated by repeated elements frequently found in human cells. Samples may furthermore be contaminated by airborne bacteria or other eukaryotes, ingested food, or symbionts living within or attached to the target organism.

The detection of contaminants in genome datasets may be accomplished pre-assembly, post-assembly, or using a combination of both approaches. Pre-assembly removal of potential contaminants has the advantage of reducing the complexity of the assembly process by producing smaller and more homogenous data sets. A first step may be filtering according to kmer-coverage or according to per-read guanine-cytosine (GC) contents (Schmieder \& Edwards, 2011) or applying more advanced binning techniques based on oligonucleotide composition (e.g. Teeling et al., 2004; McHardy et al., 2007). This bears the risk of also removing genomic sequences from the target organism e.g. repeated elements or regions resulting from recent horizontal gene transfers, and of missing contaminants with similar properties as the target genome. A complementary approach is to search for potential contaminants based on sequence similarity. Here the main limitation lies in the quality and completeness of the reference databases. Furthermore, sequence similarity searches may be time-consuming due to the amount of raw data to treat and are classically limited to smaller sets of contaminants such as vectors or individual species (e.g. to screen for human DNA contaminants), or ribosomal sequences. However, the recent development of fast search and classification algorithms that do not rely on marker genes and are dedicated to the analysis of (mainly microbial) metagenomes, such as RITA (MacDonald, Parks \& Beiko, 2012), KRAKEN (Wood \& Salzberg, 2014), or Kaiju (Menzel, Ng \& Krogh, 2016) (see Sangwan, Xia \& Gilbert, 2016 for a recent review), could also be applied to standard genome datasets with the aim of identifying contaminations prior to assembly. Just as for kmer- and GC contents-based approaches the 
59

60

61

62

63

64

65

66

67

68

69

limitation remains that foreign sequences recently integrated into the host genome, may be falsely removed.

Post-assembly cleaning of genomic sequences is frequently performed once manual annotation reveals the presence of contaminants (e.g. Cock et al., 2010; Olsen et al., 2016), and does not impact noncontaminated scaffolds. As for pre-assembly approaches, criteria to remove sequences are properties such as GC contents and read coverage (e.g. Kumar et al., 2013) or sequence similarity (e.g. Cock et al., 2010; Collén et al., 2013; Olsen et al., 2016). As mentioned above, GC- and coverage-based approaches will fail to detect contaminants that resemble the target organism with respect to these characteristics, while the performance of sequence-similarity based approaches is strongly dependent on the quality of the reference database. A key advantage of post-assembly approaches, however, is that they allow for the detection of recent horizontal gene transfer events as long as these elements are embedded into scaffolds containing also host DNA.

Here we used a simple analysis pipeline based on multiple similarity searches to detect potential contaminating sequences in the published and assembled genome of the kelp species Saccharina latissima. We show that, in our case, the approach of splitting scaffolds into small fragments prior to blast searches resulted in the identification of several hybrid scaffolds and of approximately $8 \mathrm{Mbp}$ of contaminant bacterial sequences that had previously been missed.

\section{Methods}

Two approaches are frequently used for the sequence-similarity based detection of potential contaminants: One is to perform searches with nucleotide sequences (either as blastn against a nucleotide database or blastx against a protein database), but if such searches are carried out with the entire scaffolds, in our experience, they may be biased by highly conserved regions, which frequently have very little discriminatory power (transposons, virus insertions etc.). Alternatively, protein-based searches may be performed with all predicted proteins of a genome against a reference database (frequently the NCBI non-redundant (nr) protein database or uniref90). Based on these results, scaffolds for which most proteins have best hits with expected contaminants are removed. The advantage of the protein-based approach is that, if there are several predicted proteins on a scaffold, then all of them can be analyzed independently, and the results combined to reach a conclusion. Furthermore, protein sequences are more conserved than nucleotide sequences thus making it easier to find hits. The main disadvantage, however, is that this approach depends on the available protein predictions. Not all DNA encodes proteins, and especially bacterial contaminations in eukaryote genomes frequently lack protein predictions. This lack can be partially explained by the heavy reliance of gene calling software on RNAseq data, which, in turn usually includes PolyA-selection during library preparation, removing bacterial messenger RNA from the final dataset.

Here we developed and used a pipeline called taxoblast that constitutes a compromise between both approaches. We worked with nucleotides to eliminate the impact of protein predictions, but in a first step (Figure 1) each scaffold of the $S$. latissima genome was split into small fragments of e.g. 500 base pairs (bp). Then, each of these fragments was compared individually to a reference database. This way 
each sequence fragment had the same weight in the analysis, i.e. analyses were not biased towards few highly conserved elements as may have been the case for blast searches with a single long query sequence. Internal tests aiming to identify bacterial sequences in algal genomes have exhibited very little differences between blastx searches against the NCBI protein nr (non-redundant) database and blastn/discontinuous megablast searches against the NCBI nucleotide nt database, except that blastx searches were significantly slower. Regular (continuous) megaBLAST searches are designed specifically for highly similar sequences with > 95\% sequence identity (McGinnis \& Madden, 2004) and were not sufficiently sensitive to detect potential bacterial contaminants in the genome of brown algae; this algorithm, may, however, be preferable when attempting to detect well-known contaminants such as human DNA. In a third step, the taxa corresponding to the best blast hits were summarized for each scaffold to identify potential foreign DNA fragments (note that the taxonomic resolution used in our pipeline can range from specific species to entire domains). As a rule of thumb, we considered that sequences with $>90 \%$ of hits with potential contaminants were likely to be contaminants, whereas scaffolds with $>90 \%$ of hits belonging to the target group were most likely not.

\section{Results and Discussion}

Taxoblast was used to screen the published genome of $S$. japonica for potential traces of foreign DNA. This genome had already been treated post-assembly to remove bacterial contamination using blastx searches of entire scaffolds against the NCBI nr protein database in combination with analyses of ORF density and GC contents (Ye et al., 2015). Only scaffolds $>2 \mathrm{~kb}$ were considered for our analyses, and blastn searches were carried out against the NCBI nucleotide $(n t)$ database (version July 19 ${ }^{\text {th }}, 2016$ ) with an e-value cutoff of 0.01 . Please note that decreasing this cutoff to $1 \mathrm{e}-5$ had virtually no impact on the results obtained.

The total calculation time for the blast analyses on the cluster of the "Analysis Bioinformatics for Marine Science" (ABIMS) platform (http://abims.sb-roscoff.fr) was 5 days. The other steps of the analysis took less than $10 \mathrm{~min}$ on a $2.8 \mathrm{GHz}$ Intel Xenon desktop computer. This reasonable total runtime was achieved although the megablast algorithm used in our pipeline is 909 times slower than Kraken and 83 times slower than MetaPhIAn (Wood \& Salzberg, 2014), mainly because our pipeline analyzes only the assembled genome data rather than running on raw reads. Taxonomic summaries were calculated first with the aim of distinguishing eukaryote (taxon 2759) from bacterial (taxon 2) sequences, and in a second round to distinguish diatom (taxon 2836) from brown algal (taxon 2870) sequences. Both bacteria and diatoms are known to form algal biofilms (Lage \& Graça, 2016) and are thus common contaminants in cultures of marine algae. Despite previous cleaning efforts undertaken prior to the publication of the S. japonica genome, several bacterial or bacteria-like sequences were found in the published assembly (see Supplemental Information File 1), but we did not identify any potential diatom sequences.

In total, 894 scaffolds $>2 \mathrm{kbp}$ (of 6,985) corresponding to $7.96 \mathrm{Mbp}$ of sequence information were identified as bacterial based on the $90 \%$ bacterial hits threshold defined above (Figure 2A). Please note that only 148 of these 894 scaffolds were predicted to comprise protein coding sequences (201 proteins in total), underlining the usefulness of blastn searches rather than blastp or blastx searches, at least in 
136

some cases. Nine of the scaffolds classified as bacterial contained $16 \mathrm{~S}$ fragments and could be assigned to different bacterial taxa using RDP classifier (Wang et al., 2007). These were Haliea (scaffold3770), Methylophaga (scaffold4403), an unclassified Gammaproteobacterium (scaffold6634), Lewinella (scaffold4968), an unclassified Proteobacterium (scaffold4223), Marinoscillum (scaffold3608), Pseudonocardia (scaffold7323), an unclassified Alphaproteobacterium (scaffold5114), and Flexibacter (scaffold4987). We also manually examined the five longest potential bacterial sequences without $16 \mathrm{~S}$ and confirmed the automatic classification: For scaffold2350 (50 kbp) best hits were found with Rhodobacteraceae; scaffold2282 (54 kbp) was found to be most similar to known sequences of Actinobacteria; scaffold2573 (40 kbp) contained several conserved regions with sequences from Planctomycetes; scaffold2615 (39 kbp) was clearly part of a Methylophaga genome (also detected by RDP classifier, see above); and scaffold2647 (37 kbp) had best hits with genomes of different Alphaproteobacteria. Finally, we examined the distribution of GC contents for all 894 scaffolds classified as bacterial and compared it to that of the remaining algal and unclassified scaffolds using the prinseq server version 0.20.4 (Schmieder \& Edwards, 2011) (Figure 2B). While the algal reads exhibited a unimodal distribution with a narrow peak in GC contents at ca. $49 \%$, reads classified as bacterial had a wide multimodal distribution with peaks at 30, 38, 53, and 64\% GC. This supports the hypothesis of diverse phylogenetic origins of the scaffolds classified as bacterial (Kumar et al., 2013).

Taxoblast also highlighted 1,060 scaffolds (corresponding to a total of $82 \mathrm{Mbp}$ of sequence information) that could not be attributed to either bacteria or eukaryotes with $\geq 90 \%$ of the best blast hits. These scaffolds may comprise assembly artifacts, for instance contaminating sequences that have been assembled with sequences of the target species, as well as scaffolds with insertions resulting from recent horizontal gene transfers. For the purpose of illustration we have selected the two scaffolds with the highest numbers of blast hits from this category, and manually examined them.

The first potential "hybrid" scaffold, scaffold159, is approximately $500 \mathrm{kbp}$ long. 557 blast hits were obtained for the individual 500 bp-segments of this scaffold, $26 \%$ of which (towards the 5 ' end of the sequence) were with bacteria. Consequently, we uploaded scaffold159 to the GC-Profile server (Gao \& Zhang, 2006), which searches for variations in the GC contents within a sequence, revealing one segmentation point in the scaffold: bases 1 to 79743 exhibited an average GC content of $40.7 \%$ while for bases 79,744 to 504,453 the average GC content was $49.9 \%$. Manual examination of both parts of the scaffold separately confirmed that the first part was highly similar to a cyanobacterial genome of the genus Stanieria (79\% sequence identity in aligned regions), whereas the second segment aligned with genomic sequences of the brown alga Ectocarpus siliculosus. For the second examined scaffold, scaffold248 with a total length of $422 \mathrm{kbp}$, the situation was similar, except that the segmentation point was found at position 151,859 and that the first part of the sequence was highly similar to published genomes of bacteria belonging to the Flammeovirgaceae. The corresponding GC contents were $51.6 \%$ for the first (bacterial) part of the sequence and $50.3 \%$ for the rest.

Interestingly, neither of the two examined scaffolds contained undefined bases (Ns) between the bacteria-like parts and those conserved with other brown algae, but more information would be required to distinguish between recent horizontal gene transfers and assembly errors. In particular, differences in sequencing coverage between different parts of the scaffold could provide an indication 
176 for the latter, but unfortunately, no genome browser is available for S. latissima yet. In the case of 177 similar coverage, ultimately polymerase chain reactions (PCRs) would be required to confirm horizontal

178 transfers. Although not all of the results obtained via our pipeline are as clear as these examples, the 179 presented data demonstrate that the current version of the S. japonica genome still contains substantial 180 amounts of bacterial contamination. We therefore suggest removing at least the 864 scaffolds with $181 \geq 90 \%$ bacterial hits from the genome prior to further analyses.

\section{Conclusions}

183 We used a simple analysis pipeline based on multiple blastn searches with small sequence fragments to detect sequences of different phylogenetic origins in the published genome of the kelp S. latissima. This procedure highlighted several contaminating bacterial sequences as well as hybrid scaffolds that should not or only partially be considered in future analyses of this genome. To facilitate similar analyses in other organisms, we have added a graphical user interface to our pipeline and made it publicly available at http://sdittami.altervista.org/taxoblast and via SourceForge. The output format of this pipeline is a tab-separated text file compatible with most spreadsheet programs. This makes it easy to combine results with other sources of information (e.g. GC content or coverage) or integrated alignmentfree tools such as the recently published PhylOligo (Mallet et al., 2017). Sequences requiring further attention such as the aforementioned hybrid scaffolds can be easily identified and further investigated either manually or using semi-automatic pipelines such as PhyloGena (Hanekamp et al., 2007). Currently an important limitation of our approach is that for larger (i.e. eukaryote) genomes, blast searches still need to be run on a dedicated blast server. One possibility will be to replace BLAST by recent and accelerated algorithms such as PLAST (Nguyen \& Lavenier, 2009), HS-BLASTN (Chen et al., 2015), or NSimScan (Novichkov et al., 2016) that are able to output the standard tabular BLAST format. Although the usefulness of our pipeline in other systems, e.g. for more closely related contaminant genomes remains to be tested, the presented case of the $S$. japonica genome underlines the importance of systematically including post-assembly pipelines for the detection of contaminant sequences in genome projects.

202

203

204

205

206

207

208

209

210

211

\section{Acknowledgements:}

We would like to thank the ABIMS platform (http://abims.sb-roscoff.fr) for access to their computing facilities and $\mathrm{C}$. Boyen for critical reading of the manuscript.

\section{Additional Information}

Data Availability: TaxoBlast (including the source code) is freely available at http://sdittami.altervista.org/taxoblast and via SourceForge (https://sourceforge.net/projects/taxoblast/). The S. japonica genome analyzed is available in Genbank under accession number JXRI00000000.1 (Ye et al., 2015).

\section{References}

Chen Y., Ye W., Zhang Y., Xu Y. 2015. High speed BLASTN: an accelerated MegaBLAST search tool. Nucleic 
212

213

214

215

216

217

218

219

220

221

222

223

224

225

226

227

228

229

230

231

232

233

234

235

236

237

238

239

240

241

242

243

244

245

246

247

248

249

250

251

252

253

254

255

Acids Research 43:7762-7768. DOI: 10.1093/nar/gkv784.

Cock JM., Sterck L., Rouzé P., Scornet D., Allen AE., Amoutzias G., Anthouard V., Artiguenave F., Aury JM., Badger JH., Beszteri B., Billiau K., Bonnet E., Bothwell JH., Bowler C., Boyen C., Brownlee C., Carrano CJ., Charrier B., Cho GY., Coelho SM., Collén J., Corre E., Da Silva C., Delage L., Delaroque N., Dittami SM., Doulbeau S., Elias M., Farnham G., Gachon CMM., Gschloessl B., Heesch S., Jabbari K., Jubin C., Kawai H., Kimura K., Kloareg B., Küpper FC., Lang D., Le Bail A., Leblanc C., Lerouge P., Lohr M., Lopez PJ., Martens C., Maumus F., Michel G., Miranda-Saavedra D., Morales J., Moreau H., Motomura T., Nagasato C., Napoli CA., Nelson DR., Nyvall-Collén P., Peters AF., Pommier C., Potin P., Poulain J., Quesneville H., Read B., Rensing SA., Ritter A., Rousvoal S., Samanta M., Samson G., Schroeder DC., Ségurens B., Strittmatter M., Tonon T., Tregear JW., Valentin K., von Dassow P., Yamagishi T., Van de Peer Y., Wincker P. 2010. The Ectocarpus genome and the independent evolution of multicellularity in brown algae. Nature 465:617-21. DOI: 10.1038/nature09016.

Collén J., Porcel B., Carré W., Ball SG., Chaparro C., Tonon T., Barbeyron T., Michel G., Noel B., Valentin K., Elias M., Artiguenave F., Arun A., Aury J-M., Barbosa-Neto JF., Bothwell JH., Bouget F-Y., Brillet L., Cabello-Hurtado F., Capella-Gutiérrez S., Charrier B., Cladière L., Cock JM., Coelho SM., Colleoni C., Czjzek M., Da Silva C., Delage L., Denoeud F., Deschamps P., Dittami SM., Gabaldón T., Gachon CMM., Groisillier A., Hervé C., Jabbari K., Katinka M., Kloareg B., Kowalczyk N., Labadie K., Leblanc C., Lopez PJ., McLachlan DH., Meslet-Cladiere L., Moustafa A., Nehr Z., Nyvall Collén P., Panaud O., Partensky F., Poulain J., Rensing SA., Rousvoal S., Samson G., Symeonidi A., Weissenbach J., Zambounis A., Wincker P., Boyen C. 2013. Genome structure and metabolic features in the red seaweed Chondrus crispus shed light on evolution of the Archaeplastida. Proceedings of the National Academy of Sciences of the United States of America 110:5247-52. DOI: 10.1073/pnas.1221259110.

Gao F., Zhang C-T. 2006. GC-Profile: a web-based tool for visualizing and analyzing the variation of GC content in genomic sequences. Nucleic Acids Research 34:W686-91. DOI: 10.1093/nar/gkl040.

Hanekamp K., Bohnebeck U., Beszteri B., Valentin K. 2007. PhyloGena--a user-friendly system for automated phylogenetic annotation of unknown sequences. Bioinformatics 23:793-801. DOI: 10.1093/bioinformatics/btm016.

Kumar S., Jones M., Koutsovoulos G., Clarke M., Blaxter M. 2013. Blobology: exploring raw genome data for contaminants, symbionts and parasites using taxon-annotated GC-coverage plots. Frontiers in Genetics 4:237. DOI: 10.3389/fgene.2013.00237.

Lage OM., Graça AP. 2016. Biofilms: An Extra Coat on Macroalgae. In: Algae - Organisms for Imminent Biotechnology. InTech,. DOI: 10.5772/63053.

Longo MS., O'Neill MJ., O'Neill RJ. 2011. Abundant human DNA contamination identified in non-primate genome databases. PloS One 6:e16410. DOI: 10.1371/journal.pone.0016410.

MacDonald NJ., Parks DH., Beiko RG. 2012. Rapid identification of high-confidence taxonomic assignments for metagenomic data. Nucleic Acids Research 40:e111-e111. DOI: 10.1093/nar/gks335.

Mallet L., Bitard-Feildel T., Cerutti F., Chiapello H. 2017. PhylOligo: a package to identify contaminant or untargeted organism sequences in genome assemblies. Bioinformatics in press. DOI: 10.1093/bioinformatics/btx396.

McGinnis S., Madden TL. 2004. BLAST: at the core of a powerful and diverse set of sequence analysis tools. Nucleic acids research 32:W20-5. DOI: 10.1093/nar/gkh435.

McHardy AC., Martín HG., Tsirigos A., Hugenholtz P., Rigoutsos I. 2007. Accurate phylogenetic 
256

257

258

259

260

261

262

263

264

265

266

267

268

269

270

271

272

273

274

275

276

277

278

279

280

281

282

283

284

285

286

287

288

289

290

291

292

293

294

classification of variable-length DNA fragments. Nature Methods 4:63-72. DOI: 10.1038/nmeth976.

Menzel P., Ng KL., Krogh A. 2016. Fast and sensitive taxonomic classification for metagenomics with Kaiju. Nature Communications 7:11257. DOI: 10.1038/ncomms11257.

Nguyen VH., Lavenier D. 2009. PLAST: parallel local alignment search tool for database comparison. BMC Bioinformatics 10:329. DOI: 10.1186/1471-2105-10-329.

Novichkov V., Kaznadzey A., Alexandrova N., Kaznadzey D. 2016. NSimScan: DNA comparison tool with increased speed, sensitivity and accuracy. Bioinformatics 32:2380-2381. DOI: 10.1093/bioinformatics/btw126.

Olsen JL., Rouzé P., Verhelst B., Lin Y-C., Bayer T., Collen J., Dattolo E., De Paoli E., Dittami S., Maumus F., Michel G., Kersting A., Lauritano C., Lohaus R., Töpel M., Tonon T., Vanneste K., Amirebrahimi M., Brakel J., Boström C., Chovatia M., Grimwood J., Jenkins JW., Jueterbock A., Mraz A., Stam WT., Tice H., Bornberg-Bauer E., Green PJ., Pearson GA., Procaccini G., Duarte CM., Schmutz J., Reusch TBH., Van de Peer Y. 2016. The genome of the seagrass Zostera marina reveals angiosperm adaptation to the sea. Nature 530:331-335. DOI: 10.1038/nature16548.

Sangwan N., Xia F., Gilbert JA. 2016. Recovering complete and draft population genomes from metagenome datasets. Microbiome 4:8. DOI: 10.1186/s40168-016-0154-5.

Schmieder R., Edwards R. 2011. Quality control and preprocessing of metagenomic datasets. Bioinformatics (Oxford, England) 27:863-4. DOI: 10.1093/bioinformatics/btr026.

Teeling H., Waldmann J., Lombardot T., Bauer M., Glöckner F. 2004. TETRA: a web-service and a standalone program for the analysis and comparison of tetranucleotide usage patterns in DNA sequences. BMC Bioinformatics 5:163. DOI: 10.1186/1471-2105-5-163.

Wang Q., Garrity GM., Tiedje JM., Cole JR. 2007. Naive Bayesian classifier for rapid assignment of rRNA sequences into the new bacterial taxonomy. Applied and environmental microbiology 73:5261-7. DOI: 10.1128/AEM.00062-07.

Wood DE., Salzberg SL. 2014. Kraken: ultrafast metagenomic sequence classification using exact alignments. Genome biology 15:R46. DOI: 10.1186/gb-2014-15-3-r46.

Ye N., Zhang X., Miao M., Fan X., Zheng Y., Xu D., Wang J., Zhou L., Wang D., Gao Y., Wang Y., Shi W., Ji P., Li D., Guan Z., Shao C., Zhuang Z., Gao Z., Qi J., Zhao F. 2015. Saccharina genomes provide novel insight into kelp biology. Nature communications 6:6986. DOI: 10.1038/ncomms7986.

\section{Figure legends}

Figure 1: Overview of the Taxoblast pipeline (A), the corresponding graphical user interface (B) and the generated output (C).

Figure 2: Application of the Taxoblast pipeline to identify potential bacterial sequences in the published S. japonica genome (Ye et al., 2015). (A) shows the percentage of bacterial/eukaryote blast hits over the 6731 scaffolds $>2 \mathrm{kbp}$ with blast hits ( 254 scaffolds $>2 \mathrm{kbp}$ had no hits). Dotted lines show the $90 \%$ cutoff proposed to consider a sequence as "contaminant". (B) and (C) illustrate the different distribution of GC contents in the sequences considered bacterial, and those considered eukaryotic or unclassified. 
295 Supplemental Information

296 Supplemental Information File 1: Taxoblast output when run with the S. japonica genome (scaffolds $>2$

$297 \mathrm{kbp}$ ) to distinguish between eukaryote and bacterial sequences.

298 
Figure 1

\section{Overview of the Taxoblast pipeline}

\section{(A), the corresponding graphical user interface (B) and the generated output (C).}

A

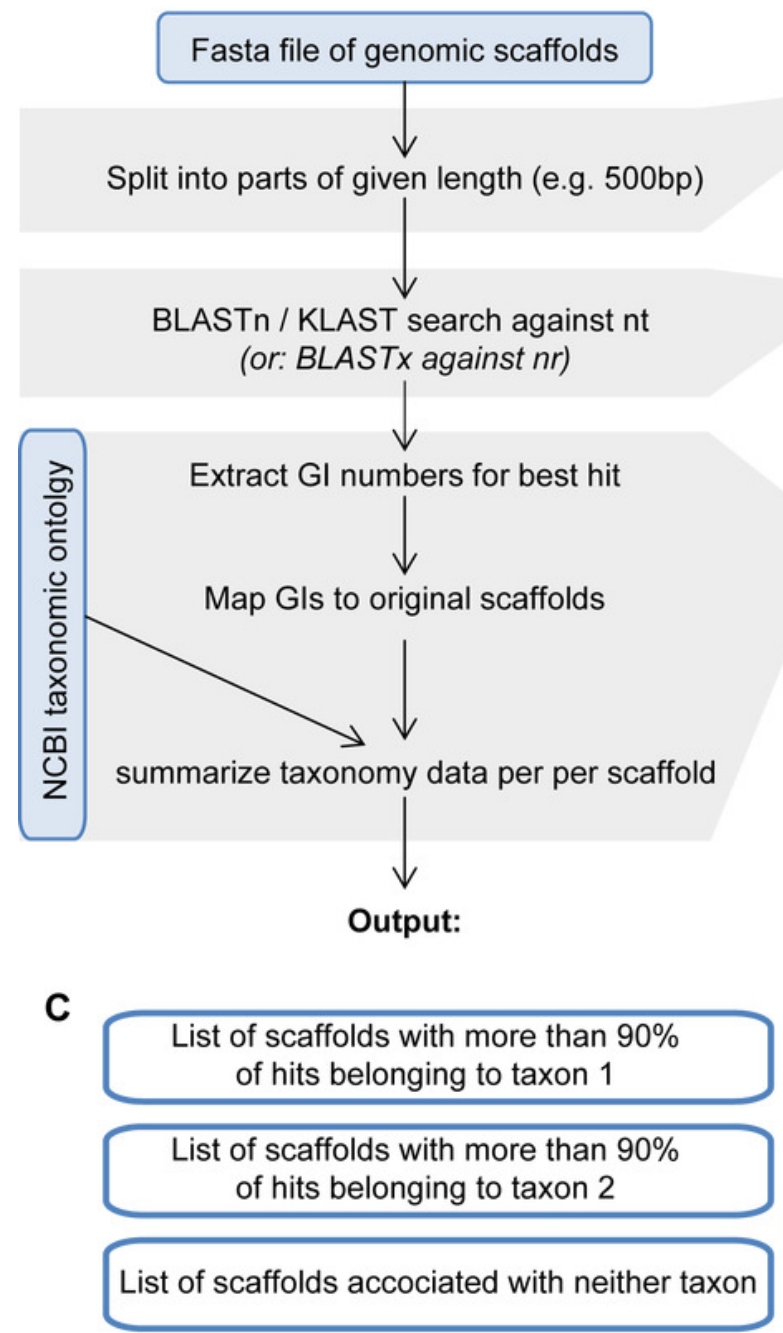

B

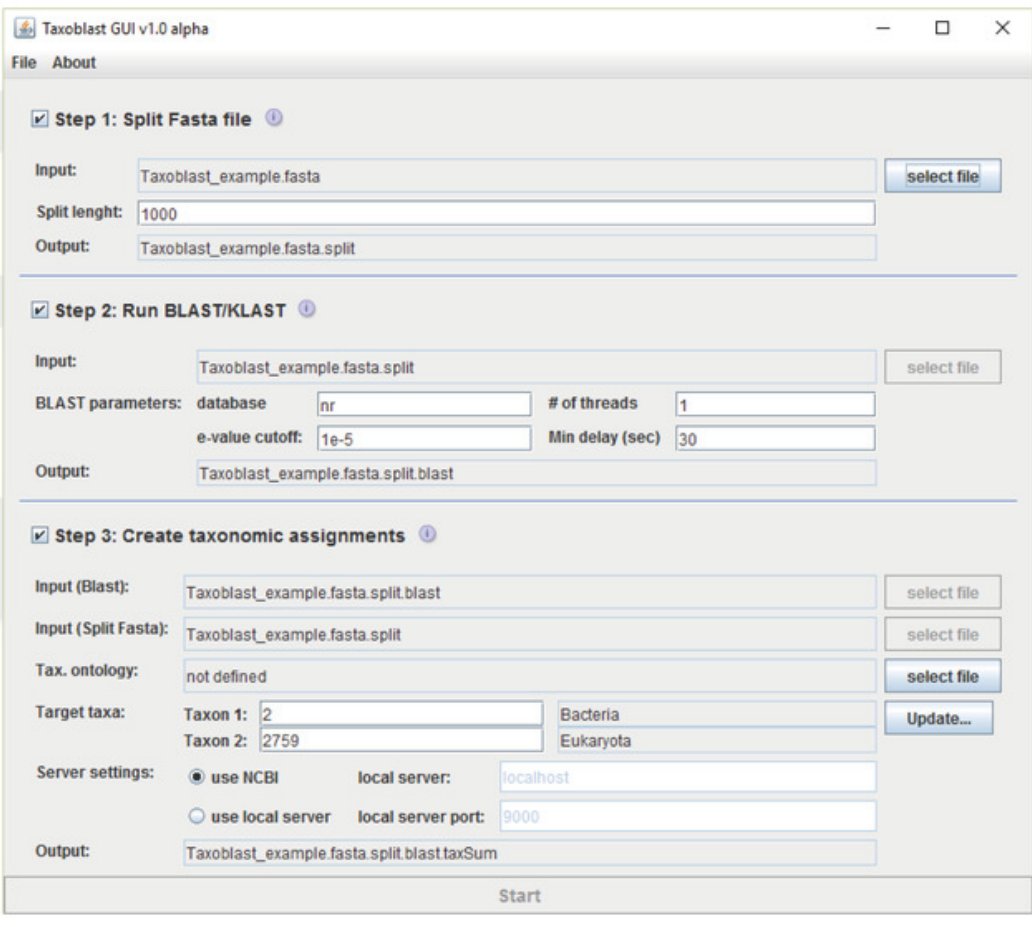

Table of results per taxon for manual fitlering Scaffold

Saccharina_japonica_cultivar_Ja_scaffold 2065 Saccharina_japonica_cultivar_Ja_scaffold5519 Saccharina_japonica_cultivar_Ja_scaffold 6435 Saccharina_japonica_cultivar_Ja_scaffold 1407 Saccharina_japonica_cultivar_Ja_scaffold6825 Saccharina_japonica_cultivar_Ja_scaffold 2815

\begin{tabular}{|r|r|r|r|r|}
\hline \#Hits & \#Queries & \%Tax:2 & \multicolumn{1}{c|}{ \%Tax:2759 } \\
\hline 45 & 133 & 2 & 97 \\
\hline 2 & 13 & 100 & 0 \\
\hline 5 & 9 & 100 & 0 \\
\hline 112 & 237 & 0 & 97 \\
\hline 4 & 7 & 100 & 0 \\
\hline 11 & 64 & 0 & 100 \\
\hline
\end{tabular}


Figure 2 (on next page)

Taxoblast analysis of the Saccharina japonica genome

Application of the Taxoblast pipeline to identify potential bacterial sequences in the published S. japonica genome (Ye et al., 2015). (A) shows the percentage of bacterial/eukaryote blast hits over the 6731 scaffolds $>2 \mathrm{kbp}$ with blast hits (254 scaffolds > $2 \mathrm{kbp}$ had no hits). Dotted lines show the $90 \%$ cutoff proposed to consider a sequence as "contaminant". (B) and (C) illustrate the different distribution of GC contents in the sequences considered bacterial, and those considered eukaryotic or unclassified. 
A

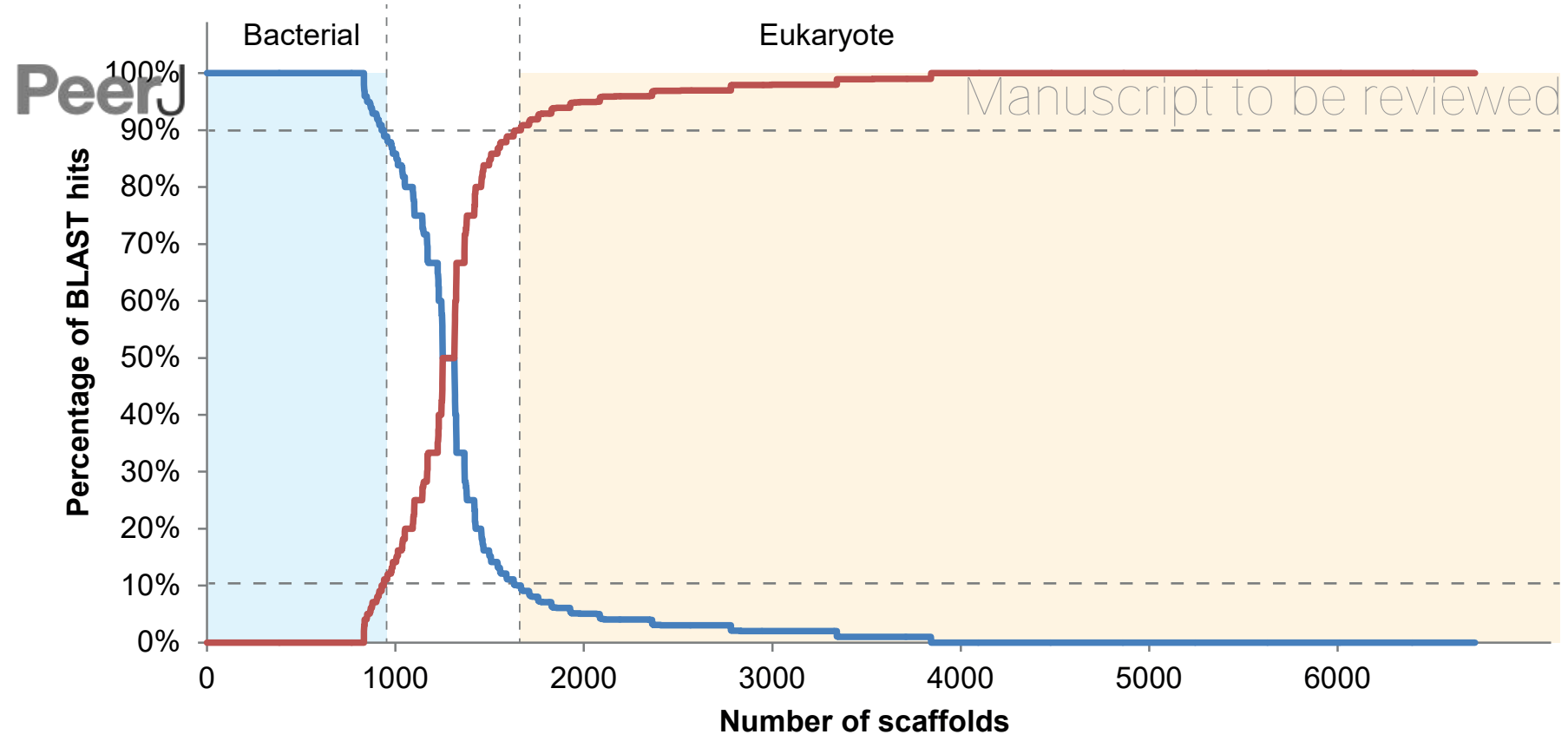

B

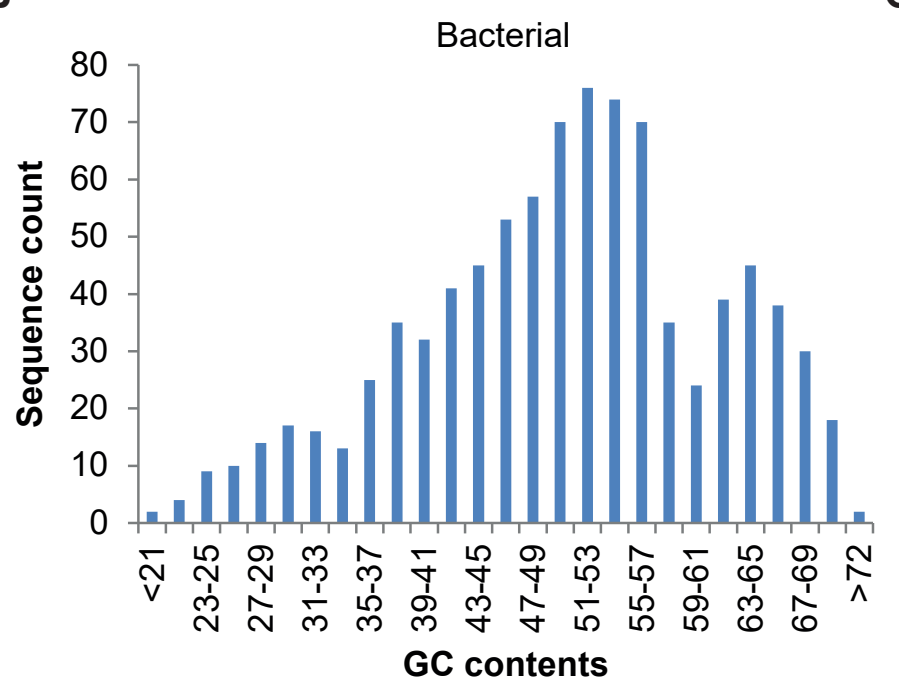

C

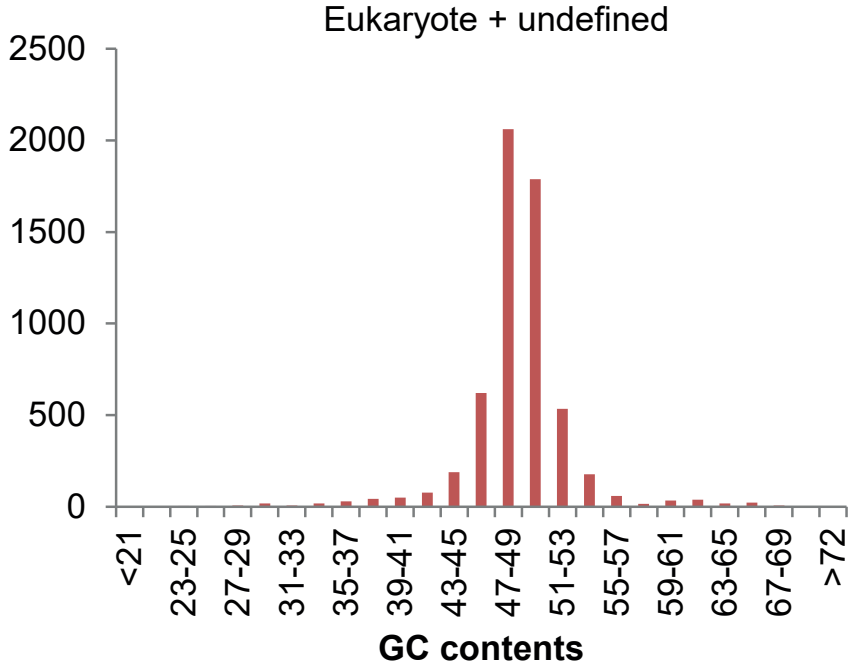

